\title{
Corrigendum: Genome-wide analysis of DNA methylation, copy number variation, and gene expression in monozygotic twins discordant for primary biliary cirrhosis
}

\section{Carlo Selmi ${ }^{1,2}{ }^{*}$, Francesca Cavaciocchi ${ }^{1,3}$, Ana Lleo ${ }^{4}$, Cristina Cheroni $^{5}$, Raffaele De Francesco ${ }^{5}$, Simone A. Lombardi ${ }^{1}$, Maria De Santis ${ }^{1,3}$, Francesca Meda ${ }^{1}$, Maria Gabriella Raimondo ${ }^{1}$, Chiara Crotti ${ }^{1}$, Marco Folci ${ }^{1}$, Luca Zammataro ${ }^{1}$, Marlyn J. Mayo ${ }^{6}$, Nancy Bach ${ }^{7}$, Shinji Shimoda ${ }^{8}$, Stuart C. Gordon ${ }^{9}$, Monica Miozzo ${ }^{10,11}$, Pietro Invernizzi ${ }^{4}$, Mauro Podda ${ }^{1}$, Rossana Scavelli ${ }^{5}$, Michelle R. Martin ${ }^{12}$, Michael F. Seldin ${ }^{13,14}$, Janine M. LaSalle ${ }^{12}$ and M. Eric Gershwin ${ }^{2}$}

${ }^{1}$ Division of Rheumatology and Clinical Immunology, Humanitas Clinical and Research Center, Milan, Italy

${ }^{2}$ Division of Rheumatology, Allergy, and Clinical Immunology, University of California at Davis, Davis, CA, USA

${ }^{3}$ BIOMETRA Department, University of Milan, Milan, Italy

${ }^{4}$ Liver Unit and Center for Autoimmune Liver Diseases, Humanitas Clinical and Research Center, Milan, Italy

${ }^{5}$ National Institute of Molecular Genetics (INGM), Milan, Italy

${ }^{6}$ University of Texas Southwestern, Dallas, TX, USA

${ }^{7}$ Mt. Sinai University, NewYork, NY, USA

${ }^{8}$ Clinical Research Center, National Nagasaki Medical Center, Nagasaki, Japan

${ }^{9}$ Henry Ford Hospital, Detroit, MI, USA

${ }^{10}$ Department of Pathophysiology and Transplantation, University of Milan, Milan, Italy

${ }^{11}$ Division of Pathology, Fondazione IRCCS Cà Granda Ospedale Maggiore Policlinico, Milan, Italy

12 Genome Center and M.I.N.D. Institute, University of California at Davis, Davis, CA, USA

${ }^{13}$ Department of Biochemistry and Molecular Medicine, University of California at Davis, Davis, CA, USA

${ }^{14}$ Department of Internal Medicine, University of California at Davis, Davis, CA, USA

*Correspondence: carlo.selmi@unimi.it

Edited and reviewed by:

Paola Zanovello, University of Padova, Italy

Keywords: autoimmune cholangitis, epigenetics, environment, DNA methylation, PBC

\section{A corrigendum on}

Genome-wide analysis of DNA methylation, copy number variation, and gene expression in monozygotic twins discordant for primary biliary cirrhosis by Selmi C, Cavaciocchi F, Lleo A, Cheroni $C$, De Francesco R, Lombardi SA, De Santis M, Meda F, Raimondo MG, Crotti C, Folci M, Zammataro L, Mayo MJ, Bach N, Shimoda S, Gordon SC, Miozzo M, Invernizzi P, Podda $M$, Scavelli $R$, Martin $M R$, Seldin MF, LaSalle JM and Gershwin ME (2014). Front. Immunol. 5:128. doi: 10.3389/fimmu.2014.00128

Author list for the article "Genome-wide analysis of DNA methylation, copy number variation, and gene expression in monozygotic twins discordant for primary biliary cirrhosis" should be as follows:

$$
\text { Carlo Selmi }{ }^{1,2^{*}} \text {, Francesca }
$$
Cavaciocchi $^{1,3}$, Ana Lleo $^{4}$, Cristina Cheroni $^{5}$, Raffaele De Francesco ${ }^{5}$, Simone A. Lombardi ${ }^{1}$, Maria De Santis ${ }^{1,3}$, Francesca Meda ${ }^{1}$, Maria Gabriella Raimondo $^{1}$, Chiara Crotti ${ }^{1}$, Marco Folci ${ }^{1}$,
Luca Zammataro ${ }^{1}$, Marlyn J. Mayo ${ }^{6}$, Nancy Bach $^{7}$, Shinji Shimoda ${ }^{8}$, Stuart C. Gordon ${ }^{9}$, Monica Miozzo ${ }^{10,11}$, Pietro Invernizzi ${ }^{4}$, Mauro Podda ${ }^{1}$, Rossana Scavelli ${ }^{5}$, Michelle R. Martin ${ }^{12}$, Michael F. Seldin ${ }^{13,14}$, Janine M. LaSalle ${ }^{12}$, and M. Eric Gershwin ${ }^{2}$

${ }^{1}$ Division of Rheumatology and Clinical Immunology, Humanitas Clinical and Research Center, Milan, Italy

${ }^{2}$ Division of Rheumatology, Allergy, and Clinical Immunology, University of California at Davis, Davis, CA, USA

${ }^{3}$ BIOMETRA Department, University of Milan, Milan, Italy

${ }^{4}$ Liver Unit and Center for Autoimmune Liver Diseases, Humanitas Clinical and Research Center, Milan, Italy

${ }^{5}$ National Institute of Molecular Genetics (INGM), Milan, Italy

${ }^{6}$ University of Texas Southwestern, Dallas, TX, USA

${ }^{7}$ Mt. Sinai University, New York, NY, USA

${ }^{8}$ Clinical Research Center, National Nagasaki Medical Center, Nagasaki, Japan

${ }^{9}$ Henry Ford Hospital, Detroit, MI, USA
${ }^{10}$ Department of Pathophysiology and Transplantation, University of Milan, Milan, Italy

${ }^{11}$ Division of Pathology, Fondazione IRCCS Cà Granda Ospedale Maggiore Policlinico, Milan, Italy

${ }^{12}$ Genome Center and M.I.N.D. Institute, University of California at Davis, Davis, CA, USA

${ }^{13}$ Department of Biochemistry and Molecular Medicine, University of California at Davis, Davis, CA, USA

${ }^{14}$ Department of Internal Medicine, University of California at Davis, Davis, CA, USA

The original article has been updated.

Conflict of Interest Statement: The authors declare that the research was conducted in the absence of any commercial or financial relationships that could be construed as a potential conflict of interest.

Received: 14 July 2014; accepted: 20 July 2014; published online: 14 August 2014

Citation: Selmi C, Cavaciocchi F, Lleo A, Cheroni C, De Francesco R, Lombardi SA, De Santis M, Meda F, Raimondo MG, Crotti C, Folci M, Zammataro L, Mayo MJ, Bach N, Shimoda S, Gordon SC, Miozzo M, Invernizzi $P$, Podda M, Scavelli R, Martin MR, Seldin MF, LaSalle JM 
and Gershwin ME (2014) Corrigendum: Genome-wide analysis of DNA methylation, copy number variation, and gene expression in monozygotic twins discordant for primary biliary cirrhosis. Front. Immunol. 5:371. doi: 10.3389/fimmu.2014.00371

This article was submitted to Inflammation, a section of the journal Frontiers in Immunology.
Copyright (C) 2014 Selmi, Cavaciocchi, Lleo, Cheroni, De Francesco, Lombardi, De Santis, Meda, Raimondo, Crotti, Folci, Zammataro, Mayo, Bach, Shimoda, Gordon, Miozzo, Invernizzi, Podda, Scavelli, Martin, Seldin, LaSalle and Gershwin. This is an openaccess article distributed under the terms of the Creative Commons Attribution License (CC BY). The use, distribution or reproduction in other forums is permitted, provided the original author(s) or licensor are credited and that the original publication in this journal is cited, in accordance with accepted academic practice. No use, distribution or reproduction is permitted which does not comply with these terms. 